\title{
Desempenho e características de carcaça de cordeiros Santa Inês confinados, filhos de ovelhas submetidas à suplementação alimentar durante a gestação
}

\author{
Marilice Zundt ${ }^{2}$, Francisco de Assis Fonseca de Macedo ${ }^{3}$, José Luís de Lima Astolphi ${ }^{4}$, \\ Alexandre Agostinho Mexia ${ }^{4}$, Eduardo Shiguero Sakaguti ${ }^{3}$
}

\footnotetext{
1 Parte da tese de doutorado em Zootecnia do primeiro autor. Pesquisa financiada pela Capes.

${ }^{2}$ Doutora em Zootecnia pela Universidade Estadual de Maringá - PR.

${ }^{3}$ Departamento de Zootecnia - Universidade Estadual de Maringá - Av. Colombo, 5790. Cep: 87020-900. Maringá-PR

${ }^{4}$ Pós-graduação em Zootecnia - Universidade Estadual de Maringá.
}

RESUMO - Objetivou-se avaliar o desempenho e as características de carcaça de cordeiros confinados, filhos de ovelhas Santa Inês submetidas à suplementação em diferentes fases da gestação. Foram inseminadas 48 ovelhas Santa Inês, com peso vivo médio de $40 \mathrm{~kg}$, distribuídas em quatro tratamentos em diferentes fases da gestação. No tratamento controle, os animais receberam somente pastagem de capim-tanzânia e resíduo de mandioca e, nos demais, houve suplementação alimentar com milho e farelo de soja, fornecidos conforme a fase de gestação (terços inicial e médio: 90:10 e 75:25). Após o desmame, os cordeiros foram confinados e receberam ração peletizada com $75 \%$ NDT e $20 \%$ PB. Ao atingirem peso vivo médio de $30 \mathrm{~kg}$, foram abatidos. A suplementação alimentar nas diferentes fases de gestação não influenciou nenhuma das variáveis estudadas e, portanto, o resíduo de mandioca pode ser fornecido às ovelhas durante toda a gestação.

Palavras-chave: características quantitativas, ganho de peso, ovinos, rendimento de carcaça

\section{Production and carcass characteristic of confined lambs born from Santa Inês ewes supplemented in different stages of pregnancy}

\begin{abstract}
This study was conducted to evaluate production and carcass characteristics of confined lambs born from Santa Inês ewes, which were supplemented in different stages of pregnancy. Forty-eight Santa Inês ewes averaging $40 \mathrm{~kg}$ of body weight were randomly assigned to one of four treatments as follows: control, animals received pasture of Panicum maximum Jacq cv. Tanzania and residue of cassava bagasse while in the remaining treatments animals were fed the same as in the control but also a supplement (corn and soybean meal) from day 1 to day 50 (treatment 2), from day 51 to day 100 (treatment 3), or from day 101 to calving (treatment 4). Proportions of corn and soybean meal in the supplement varied from 90:10 (initial and mid gestation) to 10:90 (end of gestation). After weaning, lambs received a pelleted diet containing $75 \% \mathrm{TDN}$ and $20 \% \mathrm{CP}$ and were finished in drylot. Lambs were slaughtered when they reached $30 \mathrm{~kg}$ of body weight. Supplementation of ewes with corn and soybean meal during different stages of pregnancy did not affect any of the studied variables. Therefore, residue of cassava bagasse can be fed to ewes at all gestational stages.
\end{abstract}

Key Words: carcass yield, quantitative carcass, sheep, weight gain

\section{Introdução}

O ganho de peso é uma variável importante tanto para o desempenho produtivo animal quanto para a avaliação da eficiência da dieta. O conhecimento da faixa etária em que ocorre a maior taxa de crescimento permite programar o abate para a fase em que diminui a eficiência alimentar (Silva Sobrinho, 2001). Assim, evitam-se idades muito avançadas e/ou alta deposição de gordura na carcaça, característica fundamental para o consumidor moderno, que não tolera carnes com elevadas quantidades de tecido adiposo. No entanto, um dos fatores que influenciam sobremaneira o ganho de peso é a deposição de massa muscular dos animais e, conseqüentemente, o período de sua formação.

Em ovinos, as fibras musculares começam a ser formadas em torno dos 32 dias de gestação (Wilson et al., 1992) e são denominadas fibras primárias, atingindo seu número máximo aos 38 dias. Nessa idade, são observadas as primeiras fibras secundárias, as quais são completamente formadas entre 80 e 125 dias de gestação (Ashmore et al., 1972; Swatland \& Cassens, 1973). Foi demonstrado que músculos como diafragma, gastrocnêmio medial, extensor digitório longo e sóleo estão completamente formados aos 140 dias de gestação em ovinos (Finkelstein et al., 1992). No entanto, 
o número de fibras primárias formadas durante o período embrionário é determinado geneticamente e não sofre influências ambientais, enquanto o número de fibras secundárias é susceptível a muitos fatores, inclusive a nutrição materna, que, em determinada fase da gestação, é o fator mais importante, em decorrência da variação no número de fibras ao nascimento (Wigmore \& Stickland, 1983).

A nutrição pré-natal adequada contribui para a hipertrofia das fibras primárias, resultando em hiperplasia das fibras secundárias, uma vez que as secundárias são comandadas pelas primárias. De acordo com Wilson et. al. (1992), as fibras primárias servem como "andaime" para a formação (em volta) das secundárias. Assim, a nutrição adequada da ovelha no período de formação das fibras pode proporcionar o nascimento de cordeiros com maior número de fibras musculares, o que promoveria maior desenvolvimento muscular, resultando em maior ganho de peso desses animais.

Entretanto, a nutrição materna inadequada pode reduzir o número de fibras musculares, estabelecido durante o período pré-natal (Ashmore et al., 1974; Alexander, 1974), uma vez que não ocorre hiperplasia muscular em mamíferos após o nascimento. A nutrição inadequada da ovelha durante a gestação pode limitar a capacidade de crescimento pós-natal dos músculos esqueléticos dos cordeiros (Greenwood et al., 2000).

Existem controvérsias acerca da época em que a nutrição materna inadequada interfere na formação das fibras musculares. Segundo Everity (1968), a redução do número de miofibras em fetos ocorre quando a desnutrição materna é severa nos terços inicial e final da gestação. Swatland \& Cassens (1973), no entanto, afirmaram que a redução do número de fibras musculares ocorre somente se a nutrição materna for inadequada desde o início da gestação, enquanto Nordby et al. (1987) descreveram que a desnutrição moderada das ovelhas durante o início da gestação não afeta o desenvolvimento muscular dos cordeiros.

Como o ganho de peso, o rendimento de carcaça é também uma característica importante na avaliação dos animais. O rendimento está diretamente relacionado ao valor comercial de cordeiros, pois geralmente é um dos primeiros índices a ser considerado, por expressar a relação percentual entre o peso da carcaça e o peso vivo do animal.

O rendimento de carcaça em ovinos varia de 45 a $60 \%$, podendo ser influenciado por fatores como raça, peso de abate, sistema de alimentação e idade do animal (Sañudo \& Sierra, 1986). De acordo com Pérez (1995), o rendimento de carcaça é determinante do maior ou menor custo da carne para o consumidor, tornando-se relevante para os criadores que investem nessa atividade.
$\mathrm{Na}$ avaliação de carcaças, o rendimento está sujeito a variações decorrentes da forma como é calculado. Segundo Osório et al. (1998), esta variável pode ser calculada pela relação entre o peso da carcaça fria e o peso vivo ao abate, em jejum (rendimento comercial), ou pela relação entre o peso da carcaça quente e o peso corporal vazio (rendimento verdadeiro ou biológico).

O peso e a conformação das carcaças também são cada vez mais considerados na comercialização, como critério de avaliação do seu valor (Osório et al., 1996). Conforme Osório (1992), o uso da conformação objetiva medir, indiretamente, a quantidade de carne da carcaça, permitindo avaliar, principalmente, o desenvolvimento muscular, enquanto o peso representa a totalidade dos tecidos que a compõem. Conformação adequada indica desenvolvimento proporcional das distintas regiões anatômicas que integram a carcaça, de modo que as melhores conformações são alcançadas quando as partes de maior valor comercial estão bem pronunciadas (Oliveira et al., 2002).

Os índices de compacidade da carcaça e da perna indicam a relação entre as massas muscular e adiposa e o comprimento e servem para avaliação da quantidade de tecido depositado por unidade de comprimento, representando a avaliação objetiva da conformação (Cunha et al., 2002).

Objetivou-se avaliar o desempenho e as características de carcaça de cordeiros confinados, filhos de ovelhas Santa Inês submetidas à suplementação alimentar em diferentes períodos da gestação.

\section{Material e Métodos}

Com uso de laparoscópio, foram inseminadas 48 ovelhas Santa Inês (peso médio de $40 \mathrm{~kg}$ ) com sêmem fresco de quatro reprodutores da mesma raça. Os cios dessas ovelhas foram sincronizados, utilizando-se o método hormonal (Corteel, 1994). Trinta dias após a inseminação, a prenhez foi detectada pela técnica de ultra-sonografia.

Durante o experimento, o rebanho permaneceu em pastagem de capim-tanzânia (Panicum maximum Jacq. cv. Tanzânia) no período diurno e, no período noturno, foi recolhido em instalações cobertas, com piso ripado e suspenso, tendo disponível no cocho resíduo de fecularia ( $2 \mathrm{~kg} / \mathrm{animal} / \mathrm{dia})$.

Foi estimada a disponibilidade de massa da forragem e determinadas as proporções da planta (folhas, colmos e material morto) e a composição química da folha do capim-tanzânia (Panicum maximum Jacq.), por meio do corte das plantas, feito rente ao solo. No período entre a estação de monta e o final dos nascimentos, a disponibilidade de $1.323 \mathrm{~kg} \mathrm{MS} / \mathrm{ha} / \mathrm{mês}$ foi $53 \%$ de folhas, $36 \%$ de colmos e $11 \%$ de matéria morta. 
Foram utilizados quatro tratamentos: no tratamento controle, os animais receberam somente pastagem e resíduo da indústria de fécula de mandioca durante toda a gestação e, nos demais tratamentos, houve a seguinte suplementação alimentar:

- terço inicial (TI): pastagem e resíduo de mandioca + suplementação do 10 ao $50^{\circ}$ o dia de gestação;

- terço médio (TM): pastagem e resíduo de mandioca + suplementação do $51^{\circ}$ ao $100^{\circ}$ dia de gestação;

- terço final (TF): pastagem e resíduo de mandioca + suplementação do $101^{\circ}$ dia de gestação até o parto.

O suplemento alimentar foi composto de milho e farelo de soja, fornecidos nas proporções de 90:10 (TM) e 75:25 (TF), respectivamente, de modo que atendesse à exigência nutricional das ovelhas nas diferentes fases de gestação. Segundo o NRC(1985), as exigências de ovelhas para os terços inicial e médio são de $11 \%$ de PB e $67 \%$ de NDT e, no terço final, de $17 \%$ de PB e $94 \%$ de NDT. O suplemento foi fornecido somente no período da manhã, antes de os animais irem para a pastagem, nas quantidades de $0,170 \mathrm{~kg} / \mathrm{dia}$, para os dois primeiros terços, e de $0,350 \mathrm{~kg} / \mathrm{dia}$, para o terço final da gestação. A composição química da pastagem e dos alimentos pode ser observada na Tabela 1 .

As ovelhas tiveram acesso a uma mistura de sal mineral (ad libitum) nas instalações e foram vacinadas contra carbúnculo, gangrena e enterotoxemia. A infecção por endoparasitos foi controlada, efetuando-se a everminação quando a contagem de ovos por gramas de fezes (OPG) foi superior a $500 \mathrm{OPG}$.

Após a parição das ovelhas, registraram-se o peso ao nascimento dos cordeiros (PN) algumas horas após nascimento e calcularam-se os ganhos de peso diários do nascimento ao desmame (GPND) e do desmame ao abate (GPDA).

Na primeira semana, as ovelhas permaneceram confinadas com seus cordeiros durante 24 horas e, na segunda e terceira semanas, somente as ovelhas tiveram acesso à pastagem 4 horas/dia, no período vespertino. Da quarta semana até o desmame (60 dias), as ovelhas permaneceram em pastagem durante o dia e foram recolhidas à noite. $\mathrm{Da}$ terceira semana de vida até o desmame, os cordeiros tiveram acesso a uma ração no creep feeding (comedouro seletivo), formulada para ganho de peso médio de $250 \mathrm{~g} / \mathrm{dia}$ (NRC, 1985), conforme a Tabela 2.

Os cordeiros foram vacinados contra carbúnculo, gangrena, enterotoxemia e ectima contagioso.

Após o desmame, 27 cordeiros (12 machos e 15 fêmeas) nascidos de parto simples ou duplo foram confinados em baias coletivas, cobertas, com piso ripado e suspenso, onde receberam raçãopeletizada com $75 \%$ NDTe $20 \% \mathrm{~PB}$ (Tabela 3 )e água à vontade, durante todo o período experimental. Avaliou-se o ganho de peso por animal e registraram-se os valores médios para consumo de matéria seca e conversão alimentar, visto que não foi estabelecido o consumo individual.

Os animais foram pesados no início do experimento e a cada 14 dias e as rações foram fornecidas uma vez ao dia, totalizando, em média, 4,0\% PV, de forma a proporcionar sobras diárias de aproximadamente $20 \%$. A proporção volumoso:concentrado da ração foi de 30:70.

Ao atingirem a média de $30 \mathrm{~kg}$ de PV na origem, os cordeiros foram abatidos após 18 horas sob dieta hídrica. A insensibilização foi feita por meio de descarga elétrica de $220 \mathrm{~V}$ por 8 segundos e a sangria, pela secção das veias jugulares e das artérias carótidas.

Previamente ao abate, determinou-se a condição corporal (CC) pelo método desenvolvido na Inglaterra por Russel et al. (1969), que se baseia na palpação da região dorsal da coluna vertebral, verificando-se a quantidade de gordura e músculo encontrada no ângulo formado pelos processos dorsais e transversais. Dessa forma, são atribuídos valores de 1 a 5 , em que 1 representa um animal caquético e 5 um animal obeso.

Ao abate, os animais foram novamente pesados (peso vivo ao abate). Em seguida, o aparelho gastrintestinal foi esvaziado para obtenção do peso corporal vazio (peso vivo ao abate menos o peso do conteúdo gastrintestinal), visando determinar o rendimento verdadeiro, relação entre o peso da carcaça quente e o peso corporal vazio (Sañudo \& Sierra, 1986).

Terminada a evisceração, as carcaças foram pesadas (peso da carcaça quente) e, duas horas após, transferidas para uma câmara frigorífica a $4^{\circ} \mathrm{C}$, onde permaneceram por 24 horas, penduradas pelos tendões em ganchos apropriados,

Tabela 1- Composição química dos alimentos, com base na MS Table 1 - Chemical composition of the dietary ingredients, DM basis

\begin{tabular}{|c|c|c|c|c|c|c|}
\hline $\begin{array}{l}\text { Ingrediente } \\
\text { Ingredient }\end{array}$ & $\begin{array}{c}\text { MS (\%) } \\
D M\end{array}$ & $\begin{array}{c}\mathrm{PB}(\%) \\
C P\end{array}$ & $\begin{array}{c}\text { FDN }(\%) \\
N D F\end{array}$ & $\begin{array}{c}\text { EB }(\mathrm{kcal} / \mathrm{kg}) \\
G E\end{array}$ & $\begin{array}{c}\mathrm{EE}(\%) \\
E E\end{array}$ & $\begin{array}{c}\text { Cinzas }(\%) \\
\text { Ash }\end{array}$ \\
\hline Milho (Corn grain) & 88,00 & 8,50 & 11,40 & 3,93 & 3,28 & 1,28 \\
\hline Capim-tanzânia (Panicum maximum Jacq cv Tanzania) & 25,00 & 9,00 & 64,97 & 4,10 & 1,73 & 9,70 \\
\hline Resíduo de fécula de mandioca (Residue of cassava bagasse) & 14,20 & 1,75 & 45,38 & 3,78 & 0,30 & 1,40 \\
\hline
\end{tabular}


Tabela 2 - Composições química e percentual da ração dos cordeiros no creep feeding

Table 2 - Chemical and ingredient composition of the experimental diets of lambs in creep feeding

\begin{tabular}{lc}
\hline Ingrediente & $\% \mathrm{MS}$ \\
Ingredient & $D M$ \\
\hline Milho (Corn grain) & 55,0 \\
Farelo de soja (Soybean meal) & 24,0 \\
Farelo de trigo (Wheat middlings) & 9,0 \\
Feno de aveia (Oat hay) & 10,0 \\
Mineral para ovinos (Minerals for lambs) & 2,0 \\
Lisina (Lysine) & 0,21 \\
Metionina (Methionine) & 0,075 \\
\hline
\end{tabular}

Nutriente

Nutrient

MS (\%) (DM)

$\mathrm{PB}(\%)(C P)$

EM $(\mathrm{Mcal} / \mathrm{kg})(M E)$

$\mathrm{Ca}$

91,20

20

3,0

1,46

$\mathrm{P}$

1,0

para manutenção das articulações tarsometatarsianas distanciadas em $17 \mathrm{~cm}$.

Ao final desse período, pesaram-se as carcaças frias, obtendo-se o rendimento comercial (relação entre o peso da carcaça fria e o peso vivo ao abate). Para cálculo dos índices de compacidade, foram realizadas as seguintes mensurações (Sañudo \& Sierra, 1986): comprimento da perna = distância entre o períneo e o bordo anterior da superfície articular tarsometatarsiana, tomada com fita métrica; comprimento interno da carcaça $=$ distância máxima entre o bordo anterior da sínfise ísquio-pubiana e o bordo anterior da primeira costela em seu ponto médio, tomada com fita métrica; e largura da garupa = largura máxima entre os trocânteres de ambos os fêmures, tomada com compasso e medida com fita métrica.

Foram calculados os índices de compacidade da carcaça (peso da carcaça fria dividido pelo comprimento interno da carcaça) e de compacidade da perna (largura da garupa dividida pelo comprimento da perna).

Realizou-se a avaliação subjetiva do grau de conformação das carcaças, segundo metodologia de Colomer-Rocher (1988), determinada pela avaliação visual da carcaça, considerando-a como um todo em diferentes regiões anatômicas (perna, garupa, lombo e espádua), e pela espessura de seus planos muscular e adiposo, em relação ao tamanho do esqueleto, sendo atribuídos valores 1,00 para conformação muito pobre, e 5,00 para excelente, fracionadas em 0,5.

O delineamento foi inteiramente casualizado e o modelo utilizado encontra-se abaixo:

$$
\mathrm{Y}_{i j k 1}=\mu+\mathrm{T}_{i}+\mathrm{S}_{j}+\mathrm{P}_{k}+\mathrm{TS}_{i j}+\mathrm{TP}_{i k}+\mathrm{e}_{i j k l},
$$

em que $\mathrm{y}_{i j k}$ é a observação referente ao animal $\mathrm{k}$ do sexo $\mathrm{j}$ do tratamento $\mathrm{i} ; \mu$, a constante geral; $\mathrm{T}_{i}$, o efeito do tratamento;

Tabela 3 - Composições química e percentual da ração dos cordeiros no confinamento

Table 3 - Chemical and ingredient composition of diets of feedlot lambs

Ingrediente

Ingredient

Milho (\%) (Corn grain) 35

Farelo de soja (\%) (Soybean meal) 32

Feno de tifton (\%) (Tifton hay) 30

Sal (\%) (Salt)

Melaço (\%) (Molasses)

Baccitracina de zinco (kg) (Zinc bacitracin) $\quad 0,200$

Monensina ( $\mathrm{kg}$ ) (Monesin)

Nutriente

Nutrient $\%$

MS (DM)

$\mathrm{PB}(C P)$

91,8

$\mathrm{EE}$

FDN $(N D F)$

MM (Ash)

NDT $(T D N)$

20,0

1,9

34,0

4,9

75

$\mathrm{S}_{j}$, o efeito do sexo; $\mathrm{P}_{k}$, o efeito do tipo de parto; $\mathrm{TS}_{i j}$, o efeito da interação tratamento $\times$ sexo; $\mathrm{TP}_{i k}$, o efeito da interação entre tratamento e tipo de parto; $\mathrm{e}_{i j k l}$, o erro aleatório associado a cada observação $\mathrm{y}_{i j k l}$.

Utilizaram-se o programa SAS (1996) e o procedimento GLM para as características que apresentaram distribuição normal dos erros aleatórios.

\section{Resultados e Discussão}

As médias e os erros-padrão para peso ao nascimento (PN), ganho de peso do nascimento ao desmame (GPND) e do desmame ao abate (GPDA) podem ser visualizadas na Tabela 4. A suplementação nas diferentes fases de gestação não influenciou o peso ao nascimento $(\mathrm{PN})$ e os ganhos de peso diário, do nascimento ao desmame (GPND) e do desmame ao abate (GPDA), sendo as médias gerais de $3,20 \mathrm{~kg}$, $0,170 \mathrm{~kg} / \mathrm{dia}$ e $0,175 \mathrm{~kg} / \mathrm{dia}$, respectivamente. O efeito de sexo e do tipo de parto não foi $(\mathrm{P}>0,05)$ significativo para nenhuma das variáveis estudadas, não se observando significância para as interações.

Em pesquisa realizada por Otto et al. (1997), cordeiros de parto gemelar que receberam ração no creep feeding tiveram o mesmo desempenho daqueles de parto simples até a idade de abate (quatro meses). Segundo Fernandes et al. (2001), cordeiros Morada Nova de partos múltiplos podem ter desempenhos semelhantes aos de parto simples, em decorrência do ganho de peso compensatório após o desmame, que diminui proporcionalmente as diferenças observadas ao desmame.

Quanto ao sexo, vários autores constataram similaridade para o desempenho entre machos e fêmeas (Tatum et al., 
1998; Macit et al., 2002; Phillips et al., 2002; Santos et al., 2002; Zundt et al., 2002)

Santos et al. (2002), trabalhando com reprodutores de raças de corte (Suffolk, Ile de France e Pool Dorset) em fêmeas Santa Inês (SI), verificaram os seguintes pesos ao nascimento: $\mathrm{SI}=3,6 \mathrm{~kg} ; 1 / 2$ Suffolk $=4,4 \mathrm{~kg} ; 1 / 2$ Ile de France $=3,8 \mathrm{~kg} ; \mathrm{e}^{1 / 2}$ Pool Dorset $=3,6 \mathrm{~kg}$ e observaram maior peso $(\mathrm{P}<0,01)$ para os mestiços Suffolk, seguidos dos 1/2 Ile de France. O peso ao nascimento encontrado para a raça Santa Inês foi similar ao deste trabalho.

Cunha et al. (2000) avaliaram o desempenho em confinamento de cordeiros de vários genótipos e observaram, em mestiços Suffolk x Ideal, peso ao nascimento (PN) de 4,2 kg, com GPND de 0,194 kg/dia e GPDA de 0,213 kg/dia. Nos cordeiros Suffolk x Corriedale, no entanto, o PN, GPND e GPDA foram, respectivamente, de 4,2 kg; 0,181 kg/dia e $0,206 \mathrm{~kg} / \mathrm{dia}$. Ambos os PN e GP encontrados por esses autores foram superiores aos observados nesta pesquisa, talvez por terem trabalhado com raças destinadas à produção de carne no cruzamento industrial.

Objetivando estudar o desempenho de cordeiros Suffolk criados em creep feeding, Garcia et al. (2003) verificaram peso médio ao nascimento de $5,5 \mathrm{~kg}$ e ganhos de peso médio no confinamento de $0,405 \mathrm{~kg} / \mathrm{dia}$. Segundo os autores, o bom peso ao nascimento resultou da boa condição corporal das ovelhas que reeberam suplementação alimentar no terço final da gestação.

Ribeiro et al. (2003) realizaram uma pesquisa com o objetivo de avaliar o peso ao nascimento (PN) e os ganhos de peso do nascimento ao desmame (GPND) e do desmame ao abate (GPDA). Os autores utilizaram cordeiros inteiros e castrados, filhos de reprodutores Hampshire Down, Ile de France e Suffolk com ovelhas Corriedade e verificaram PN de 3,77; 4,21 e 3,68 kg, GPND de 0,169; 0,160 e 0,138 kg/dia; e GPDA de 0,$187 ; 0,167$ e $0,204 \mathrm{~kg} /$ dia para os respectivos cruzamentos.

Em cordeiros puros Santa Inês (SI) terminados em confinamento recebendo dietas com diferentes fontes de óleo vegetal, Yamamoto et al. (2005) verificaram melhor ganho de peso do desmame ao abate $(0,258 \mathrm{~kg} / \mathrm{dia})$, corroborando os resultados obtidos por Rocha (2002), que encontrou ganho de peso médio de $0,231 \mathrm{~kg}$ /dia para cordeiros mestiços SI, e Susin et al. (2000), que observaram ganhos de $0,240 \mathrm{~kg} / \mathrm{dia}$.

Resultados semelhantes aos deste trabalho foram verificados por Garcia et al. (2000), em cordeiros SI terminados em confinamento (ganhos de $0,166 \mathrm{~kg} / \mathrm{dia}$ do desmame ao abate), e por Oliveira et al. (2003), em cordeiros das raças Bergamácia e Santa Inês terminados em confinamento $(0,165$ $\mathrm{kg}$ /dia para Santa Inês e 0,196 kg/dia para Bergamácia). Todavia, Barros et al. (2003), ao avaliarem o desempenho de cordeiros em confinamento, verificaram que os meio-sangue Somalis x SRD ganharam mais peso $(0,171 \mathrm{~kg} / \mathrm{dia})$ que os Santa Inês x SRD $(0,134 \mathrm{~kg} /$ dia $)$, mas esses ganhos foram inferiores aos encontrados neste trabalho.

Avaliando o desempenho de cordeiros da raça Morada Nova, utilizando o mesmo sistema de terminação, com dieta contendo $60 \%$ de concentrado e $40 \%$ de volumoso, Zeoula (2002) observou ganho de peso similar ao deste trabalho, de $0,172 \mathrm{~kg} / \mathrm{dia}$.

A análise estatística para as variáveis consumo de ração e conversão alimentar do desmame ao abate não foi possível, pois os cordeiros não foram terminados em baias individuais, e sim em baias coletivas, sendo apresentados apenas os valores numéricos médios de $0,980 \mathrm{~kg} / \mathrm{dia}$ para o consumo de ração, do desmame $(13,50 \mathrm{~kg})$ ao abate $(29 \mathrm{~kg})$, e 5,6 para conversão alimentar.

Os resultados obtidos para as variáveis subjetivas (condição corporal-CC e conformação da carcaça-CO) e objetivas (peso vivo ao abate-PVA; peso da carcaça quente-PCQ; peso da carcaça fria-PCF; rendimento verdadeiro da carcaça-RV; rendimento comercial da carcaça $-\mathrm{RC}$; índice de compacidade da carcaça - ICC; índice de compacidade da perna - ICP; e idade de abate - IA) são apresentados na Tabela 5.

Não se constatou efeito da suplementação das ovelhas, nas diferentes fases de gestação, para as características de carcaça estudadas, não se observando efeito do sexo, do tipo de parto $(\mathrm{P}>0,05)$ ou de sua interação para nenhuma das variáveis avaliadas.

Tabela 4 - Médias e erros-padrão para peso ao nascimento (PN), ganho de peso do nascimento ao desmame (GPNA) e do desmame ao abate (GPDA), de cordeiros Santa Inês de acordo com o tratamento das ovelhas durante a gestação

Table 4 - Means and standard errors for weight at birth (WB), daily weight gain from birth to weaning (ADGBW) and from weaning to slaughter (ADGWS) of lambs born from Santa Inês ewes that received different supplementation during pregnancy

\begin{tabular}{lccc}
\hline & & \multicolumn{2}{c}{$\begin{array}{c}\text { Tratamento } \\
\text { Treatment }\end{array}$} \\
\cline { 2 - 4 } $\begin{array}{l}\text { Variável } \\
\text { Item }\end{array}$ & $\begin{array}{c}\text { Controle } \\
\text { Control }\end{array}$ & $\begin{array}{c}\text { Terço inicial } \\
\text { From day 1 to } 50\end{array}$ & $\begin{array}{c}\text { Terço médio } \\
\text { From day 51 to 100 }\end{array}$ \\
\hline PN (kg) (WB) & $3,60 \pm 0,42$ & $2,90 \pm 0,44$ & $3,00 \pm 0,38$ \\
GPND (kg) (ADGBW) & $0,161 \pm 0,01$ & $0,164 \pm 0,01$ & $0,185 \pm 0,01$ \\
GPDA (kg) (ADGWS) & $0,165 \pm 0,01$ & $0,166 \pm 0,01$ & $0,191 \pm 0,01$ \\
\hline
\end{tabular}


Tabela 5 - Médias e erros-padrão para condição corporal (CC), conformação da carcaça (CO), peso vivo ao abate (PVA), peso da carcaça quente $(P C Q)$, peso da carcaça fria (PCF), rendimento verdadeiro da carcaça (RV), rendimento comercial da carcaça (RC), índice de compacidade da carcaça (ICC) e da perna (ICP) e idade de abate (IA) de cordeiros Santa Inês filhos das ovelhas submetidas à suplementação alimentar durante a gestação

Table 5 - Means and standard-errors for body condition score (BC), conformation(CO), slaughter weight (SW,) hot carcass weight (HCW), cold carcass weight (CCW), net carcass yield ( $\mathrm{NCY}$ ), commercial carcass yield (CCY), carcass compactness index (CCl), ham compactness index ( $\mathrm{HCl}$ ) and slaughter age (SA) of lambs born from Santa Inês ewes that received different supplementation during pregnancy

\begin{tabular}{|c|c|c|c|c|}
\hline \multirow[b]{2}{*}{$\begin{array}{l}\text { Variável } \\
\text { Variable }\end{array}$} & \multicolumn{4}{|c|}{$\begin{array}{l}\text { Tratamento } \\
\text { Treatment }\end{array}$} \\
\hline & $\begin{array}{l}\text { Controle } \\
\text { Control }\end{array}$ & $\begin{array}{l}\text { Terço inicial } \\
\text { From day } 1 \text { to } 50\end{array}$ & $\begin{array}{c}\text { Terço médio } \\
\text { From day } 51 \text { to } 100\end{array}$ & $\begin{array}{c}\text { Terço final } \\
\text { From day } 101 \text { to calving }\end{array}$ \\
\hline $\mathrm{CC} B C$ & $2,67 \pm 0,15$ & $2,56 \pm 0,13$ & $2,74 \pm 0,13$ & $2,79 \pm 0,22$ \\
\hline $\mathrm{CO} C O$ & $2,82 \pm 0,17$ & $2,74 \pm 0,14$ & $3,26 \pm 0,15$ & $2,62 \pm 0,24$ \\
\hline PVA $(\mathrm{kg})(S W)$ & $28,57 \pm 1,38$ & $28,48 \pm 1,19$ & $31,88 \pm 1,24$ & $27,56 \pm 1,96$ \\
\hline $\mathrm{RV}(\%)(N C Y)$ & $55,51 \pm 0,83$ & $57,04 \pm 0,72$ & $56,94 \pm 0,74$ & $56,53 \pm 1,18$ \\
\hline $\mathrm{RC}(\%)(C C Y)$ & $49,55 \pm 0,58$ & $50,03 \pm 0,50$ & $50,22 \pm 0,52$ & $49,36 \pm 0,83$ \\
\hline $\mathrm{ICC}(\mathrm{kg} / \mathrm{cm})$ & $0,23 \pm 0,01$ & $0,23 \pm 0,01$ & $0,25 \pm 0,01$ & $0,22 \pm 0,02$ \\
\hline $\mathrm{ICP}(\mathrm{HCI})$ & $0,37 \pm 0,01$ & $0,41 \pm 0,01$ & $0,41 \pm 0,01$ & $0,43 \pm 0,02$ \\
\hline IA (dias) ( $S A$, days) & $153 \pm 0,47$ & $154 \pm 0,47$ & $154 \pm 0,40$ & $154 \pm 0,49$ \\
\hline
\end{tabular}

Os valores médios para a condição corporal e a conformação foram, respectivamente, 2,70 e 2,96, superiores aos encontrados por Yamamoto et al. (2005), em cordeiros Santa Inês em confinamento, e inferiores aos obtidos por Zundt et al. (2003), em cordeiros $1 / 2$ Texel + 1/4 Bergamácia + 1/4 Corriedale, também em confinamento. ACC e aCO verificadas nesta pesquisa caracterizam a raça Santa Inês, que possui carcaças menos musculosas em comparação às raças selecionadas para produção de carne.

O peso vivo médio ao abate (PVA) foi de 29,4 kg. De acordo com Santos et al. (2001), a faixa de peso de abate ideal para cordeiros Santa Inês é de 15 a $35 \mathrm{~kg}$, pois, acima deste peso, ocorre deposição acentuada de tecido adiposo.

Os valores médios observados para PCQ e PCF foram de 15,10 e 14,67 kg, respectivamente, e são semelhantes aos encontrados por Cunha et al. (2002), em cordeiros SI em confinamento (15,6 kg para PCQ e 14,9 para PCF), abatidos aos 168 dias com peso de abate de $32,7 \mathrm{~kg}$. Santos et al. (2002), utilizando cordeiros Santa Inês (SI) abatidos com 28-30 kg de peso vivo médio, observaram valores de 13,3 e $12,7 \mathrm{~kg}$ para $\mathrm{PCQ}$ e $\mathrm{PCF}$, respectivamente. Os valores observados para PCQ e PCF podem ser considerados satisfatórios, pois encontram-se na faixa de preferência da maioria dos consumidores brasileiros.

$\mathrm{O}$ rendimento verdadeiro (RV), considerando todos os tratamentos, foi de $56,51 \%$ e o rendimento comercial (RC), de 49,97\%. Segundo Sañudo \& Sierra (1986), os rendimentos de carcaça variam de 40 a $60 \%$, conforme a raça, os cruzamentos e o sistema de criação. Logo, os dados desta pesquisa estão compatíveis com os descritos por esses autores.

Resultado similar ao deste trabalho foi encontrado por Siqueira et al. (2002), em cordeiros Santa Inês (RV de 55\%).
Entretanto, o RV médio verificado nesta pesquisa encontra-se acima da média se comparado aos resultados de outras pesquisas envolvendo o genótipo Santa Inês, como o de Fagundes Neto et al. (2002), que observaram rendimentos médios de 47 e $46 \%$ para RV e RC, respectivamente, e o de Rocha (2002), que constataram, para cordeiros Santa Inês, respectivos valores de 44,9 e 44\% para os rendimentos verdadeiro e comercial. No entanto, Garcia et al. (2000) verificaram $R C$ superiores aos desta pesquisa (53,1 e 53,4\%, respectivamente), em cordeiros Santa Inês puros e mestiços. Yamamoto et al. (2005), no entanto, verificaram para cordeiros SI e mestiços (Dorset x Santa Inês) RV similares (54,29 e $53,02 \%)$, respectivamente.

Em raças de corte, vários autores encontraram resultados similares e/ou superiores aos observados nesta pesquisa para os rendimentos verdadeiros e comerciais. Neres et al. (2001), em estudo com cordeiros Suffolk abatidos aos 26-28 kg de PV, observaram rendimentos verdadeiro e comercial de 58,37 e 52,45\%, respectivamente. Ribeiro et al. (2003) abateram cordeiros aos $30 \mathrm{~kg}$ de PV e constataram, para as raças Hampshire Down, Ile de France e Suffolk, RV de 56,$76 ; 56,51$ e $54,27 \%$ e RC de 54,$73 ; 54,31$ e $51,36 \%$, respectivamente. Garcia et al. (2003) avaliaram cordeiros Suffolk abatidos com $31 \mathrm{~kg}$ de PV terminados em confinamento e verificaram RV e RC médios de 58 e 51,1\%, respectivamente.

As médias para os índices de compacidade da carcaça (ICC, $\mathrm{kg} / \mathrm{cm}$ ) e de compacidade da perna (ICP) foram, respectivamente, 0,24 e 0,40. Reis et al. (2001) encontraram menor índice para compacidade da carcaça $(0,21 \mathrm{~kg} / \mathrm{cm})$ e maior para compacidade da perna $(0,45)$, em cordeiros mestiços Bergamácia x Corriedale. Cunha et al. (2000) observaram 
valor semelhante para ICC $(0,25 \mathrm{~kg} / \mathrm{cm})$, em cordeiros Ile de France x Ideal. Martins et al. (1999), avaliando dois grupos genéticos $(1 / 2$ Texel $+1 / 4$ Bergamácia $+1 / 4$ Corriedale e $1 / 2$ Texel $+1 / 4$ Hampshire $+1 / 4$ Corriedale), verificaram valor inferior para ICC $(0,22 \mathrm{~kg} / \mathrm{cm})$ e superior pra ICP $(0,51)$

Resultados superiores foram obtidos por Zundt et al. (2003), que verificaram ICC $(\mathrm{kg} / \mathrm{cm})$ de 0,27 e ICP de 0,43 , ao avaliarem cordeiros "tricross" ( $1 / 2$ Texel $+{ }^{1 / 4}$ Bergamácia $+{ }^{1 / 4}$ Corriedale) terminados em confinamento. Carvalho et al. (2002), em cordeiros Santa Inês alimentados ad libitum no confinamento e abatidos com $35 \mathrm{~kg}$ de PV, constataram valores de $0,29 \mathrm{~kg} / \mathrm{cm} \mathrm{e} 0,42$, para ICC e ICP, respectivamente. Siqueira et al. (2001), estudando cordeiros Ile de France x Corriedale, abatidos com $28 \mathrm{~kg}$ de PV, obtiveram ICC e ICP de $0,26 \mathrm{~kg} / \mathrm{cm}$ e 0,49 , respectivamente.

O ICC e ICP encontrados neste trabalho apresentaram valores expressivos, indicando boa deposição de tecido muscular por unidade de comprimento, quando comparados aos obtidos em outros trabalhos com o mesmo genótipo.

\section{Conclusões}

Não se recomenda a suplementação com milho e farelo de soja em dietas para ovelhas Santa Inês gestantes, pois não afetou os parâmetros estudados, independentemente da fase de gestação. O resíduo de mandioca, no entanto, pode ser fornecido durante toda a gestação.

Os valores observados para peso de carcaça quente e fria foram satisfatórios, pois encontram-se na faixa $(15 \mathrm{~kg})$ de preferência da maioria dos consumidores brasileiros.

Os índices de compacidade da carcaça e da perna apresentaram valores expressivos e indicam boa deposição de tecido muscular por unidade de comprimento para cordeiros da raça Santa Inês.

\section{Literatura Citada}

ALEXANDER, G. Birth weight of lambs: Influences and consequences. In: SIZE AT BIRTH. CIBA FOUNDATION SYMPOSIUM, 27., 1974, Amsterdam. Proceedings... Amsterdam: The Netherlands, 1974. p.215.

ASHMORE, C.R. Phenotypic expression of muscle fiber types and some implications to meat quality. Journal of Animal Science, v.38, p.1158-64, 1974

ASHMORE, C.R.; TOMPKINS, G.; DOERR, L. Posnatal development of muscle fiber types in domestic animals. Journal of Animal Science, v.34, p.37-41, 1972.

BARROS, N.N.; VASCONCELOS, V.R.; ARAÚJO, M.R. et al. Influência do grupo genético e da alimentação sobre o desempenho de cordeiros em confinamento. Pesquisa Agropecuária Brasileira, v.38, n.9, p.1111-1116, 2003.

COLOMER-ROCHER, F. Estudio de los parametros que definen los caracteres cuantitativos y cualitativos de las canales. In: CURSO INTERNACIONAL SOBRE PRODUCCIÓN DE CARNE Y
LECHE CON BASES EN PASTOS Y FORRAJES, 1988, La Coruna. Palestras... La Coruna: 1988. 108p.

CORTEEL, J.M. Activites oestrienne et ovulatorie de la chevrette et de la chevre: à puberté au cours de la période post partum et au fil des saisons. In: ENCONTRO NACIONAL PARA O DESENVOLVIMENTO DA ESPÉCIE CAPRINA, 3., 1994, Jaboticabal. Anais... São Paulo: 1994. p.98-121.

CUNHA, E.A.; SANTOS, L.E.; BUENO, M.S. et al. Utilização de carneiros de raças de corte para obtenção de cordeiros precoces para abate em plantéis produtores de lã. Revista Brasileira de Zootecnia, v.29, n.1, p.243-252, 2000.

CUNHA, E.A; BUENO, M.S.; SANTOS, L.E. 2002. Características de carcaças de cordeiros de raças de corte criados intensivamente. Disponível em: http:// www.cico.rj.gov.br Acesso em: 12/12/2002.

EVERIT, G.C. Prenatal development of uniparous animals with particular reference to the influence of maternal nutrition in sheep. In: LODGE, G.E. (Ed.) Growth and development of mammals. London: Lamming, 1968. 252p.

FAGUNDES NETO, J.C.; BRAGA, A.P.; BARRA, P.B. et al Substituição parcial do farelo de soja pela mistura milho/uréia sobre o rendimento de carcaça de ovinos mestiços Santa Inês In: REUNIÃO ANUAL DA SOCIEDADE BRASILEIRA DE ZOOTECNIA, 39., 2002, Recife. Anais... Recife: Sociedade Brasileira de Zootecnia, 2002. CD-ROM. Nutrição de ruminantes - 1227

FERNANDES, A.A.O; BUCHANAN, D.; SELAIVE-VILLARROEL, A.B. Avaliação dos fatores ambientais no desenvolvimento corporal de cordeiros deslanados da raça Morada Nova. Revista Brasileira de Zootecnia, v.30, n.5, p.1460-1465, 2001.

FINKELSTEIN, D.L.; ANDRIANAKIS, P.; LUFF, A.R. et al. Development changes in hindlimb muscles and diaphragm of sheep. American Journal Physiology, v.263, p.900-908, 1992.

GARCIA, C.A.; COSTA, C.; MONTEIRO, A.L.G. et al. Níveis de energia no desempenho e características da carcaça de cordeiros alimentados em creep feeding. Revista Brasileira de Zootecnia, v.32, n.6, p.1371-1379, 2003.

GARCIA, I.F.F.; PEREZ, J.R.O.; TEIXEIRA, C.J. et al. Desempenho de cordeiros Texel x Santa Inês puro, terminados em confinamentos, aleitamentos com casca de café como parte da dieta. Revista Brasileira de Zootecnia, v.29, n.2, p.564572,2000 .

GREENWOOD, P.L.; HUNT, A.S.; HERMANSON, J.W. et al. Effects of birth weight ans post natal nutrition on neonatal sheep. II. Skeletal muscle growth and development. Journal of Animal Science, v.78, p.50-61, 2000.

MACIT, M.; ESENBUGA, N.; KARAOGLU, M. Growth performance and carcass characteristics of Awassii, Morkaraman and Tushin lambs grazed on pastrue and supported with concentrade. Small Ruminant Research, v.44, p.241-246, 2002.

MARTINS, E.N.; MACEDO, F.A.F.; MACEDO, R.M.G. et al Desempenho e características quantitativas da carcaça de cordeiros mestiços texel, terminados em confinamento, com diferentes níveis de energia. In: REUNIÃO ANUAL DA SOCIEDADE BRASILEIRA DE ZOOTECNIA, 36., 1999, Porto Alegre. Anais... Porto Alegre: Sociedade Brasileira de Zootecnia, 1999. p.347.

NATIONAL RESEARCH COUNCIL - NRC. Nutrients requirements of sheep. Washington, D.C.: National Academy Press, 1985. 99p.

NERES, M.A.; MONTEIRO, A.L.G.; GARCIA, C.A. et al. Forma física da ração e pesos de abate nas características de carcaça de cordeiros em creep feeding. Revista Brasileira de Zootecnia, v.30, n.3, p.948-954, 2001.

NORDBY, D.J.; FIELD, M.L.; RILEY, M.L. et al. Effects of maternal undernutrition during early pregnancy on growth, muscle cellularity, fiber type and carcass composition in lambs. Journal of Animal Science, v.64, p.1419-1427, 1987.

OSÓRIO, J.C.S. Estudio de la calidad de canales comercializadas en el tipo ternasco segun la procedencia: bases para la mejora de dicha calidad en Brasil. Zaragoza: 
Universidade de Zaragoza, 1992. 335p. Tese (Doutorado em Veterinária) - Universidade de Zaragoza, 1992.

OSÓRIO, J.C.S.; ALFRANCA, I.S.; SAÑUDO, C. et al. Efeito da procedência sobre o peso e conformação da carcaça em cordeiros. Revista Brasileira de Zootecnia, v.25, n.6, p.1187-1194, 1996.

OSÓRIO, J.C.S.; ASTIZ, C.S.; OSÓRIO, M.T.M. et al. Produção de carne ovina alternativa para o Rio Grande do Sul. Pelotas: Universidade Federal de Pelotas, 1998. 165p.

OLIVEIRA, M.V.M.; PÉREZ, J.R.O.; ALVES. E.L. Avaliação da composição de cortes comerciais componentes corporais e órgãos internos de cordeiros confinados e alimentados com dejetos de suínos. Revista Brasileira de Zootecnia, v.31, n.3, p.1459-1468, 2002.

OLIVEIRA, M.V.M.; PÉREZ, J.R.O.; GARCIA, F.F. et al. Desempenho de cordeiros das raças Bergamácia e Santa Inês, terminados em confinamento, recebendo dejetos de suínos como parte da dieta. Revista Brasileira de Zootecnia, v.32, n.6, p.1391-1396, 2003.

OTTO,C; ANDRIGUETTO,J.L.; SÁ, J.L. et al. Efeito do creep feeding sobre a ingestão alimentar e desenvolvimento de cordeiros. In: INICIAÇÃO CIENTÍFICA DA UFPR, 5., 1997, Curitiba. Anais... Curitiba: Universidade Federal do Paraná, 1997. p.208.

PÉREZ, J.R.O. Alguns aspectos relacionados com a qualidade da carcaça e da carne ovina. In: SIMPÓSIO PAULISTA DE OVInOCUltura, 4., 1995, Campinas. Anais... Campinas: 1995. p.125-139.

PHILLIPS, W.A.; REUTER, R.R.; BROWN, M.A. et al. Growth and performace of lambs fed a finishing diet containing either Alfafa or Kenaf as the roughage source. Small Ruminant Reseach, v.46, p.75-79, 2002.

ROCHA, M.H.M. Teores de proteína bruta em dietas com alta proporção de concentrado para cordeiros confinados. Piracicaba: Escola Superior de Agricultura "Luiz de Queiroz", 2002. 73p. Dissertação (Mestrado em Agronomia) - Escola Superior de Agricultura "Luiz de Queiroz", 2002.

REIS, W.; JOBIM, C.C.; MACEDO, F.A.F. et al. Desempenho de cordeiros terminados em confinamento, consumindo silagens de grãos de milho em diferentes formas. Revista Brasileira de Zootecnia, v.30, n.2, p.525-532, 2001

RIBEIRO, E.L., SILVA, L.D.F., ROCHA, M.A. et al. Desempenho de cordeiros inteiros ou submetidos a diferentes métodos de castração abatidos aos $30 \mathrm{~kg}$ de peso vivo. Revista Brasileira Zootecnia, v.32, n.3, p.745-752, 2003

RUSSEL, A.J.F.; DONEY, J.M.; GUNN, R.G. Subjective assessment of body fat in live sheep. Journal Agricultural Science, v.72, p.451-454, 1969.

SANTOS, C.L.; PÉREZ, J.R.O; MUNIZ, J.A. et al. Desenvolvimento relativo dos tecidos ósseo muscular e adiposo dos cortes da carcaça de cordeiros Santa Inês. Revista Brasileira de Zootecnia, v.30, n.2, p.487-492, 2001

SANTOS, L.E.; BUENO, M.S.; CUNHA, E.A. 2002. Desempenho e características de carcaça de cordeiros Santa Inês cruzados com racas especializadas para corte. Disponível em: http://www.ovinosbrasil.com/trab-tec/pg-trab-tecs-009htm Acesso em: 15/12/2002.
SAÑUDO, C.; SIERRA, I. Calidad de la canal en la especie ovina. Ovino, n.11, p.127-57, 1986.

SILVA SOBRINHO, A.G. Criação de ovinos. 2.ed. Jaboticabal: Funep, 2001. 301p.

STATISTICAL ANALYSES SYSTEM - SAS. SAS user's guide statistics. v.2, version 6, 4.ed. Cary: 1996. 300p.

SIQUEIRA, E.R.; FERNANDES, S.; GUAZELLI, M. Efeito do peso ao abate sobre o crescimento e caracteres da carcaça de cordeiros Santa Inês e mestiços Bergamácia x Corriedale, terminados em confinamento. In: REUNIÃO ANUAL DA SOCIEDADE BRASILEIRA DE ZOOTECNIA, 39., 2002, Recife. Anais... Recife: Sociedade Brasileira de Zootecnia, 2002. CD ROM. Nutrição de ruminantes - 220

SIQUEIRA, E.R.; SIMOES, C.D.; FERNANDES, S. Efeito do sexo e do peso ao abate sobre a produção de carne de cordeiro. Morfometria da carcaça, pesos dos cortes, composição tecidual e componentes não-constituintes da carcaça. Revista Brasileira de Zootecnia, v.30, n.4, p.1299-1307, 2001.

SUSIN, I.; ROCHA, M.H.M.; PIRES, A.V. Efeito do uso do bagaço de cana de açúcar in natura ou hidrolisado sobre o desempenho de cordeiros confinados. In: REUNIÃO ANUAL DA SOCIEDADE BRASILEIRA DE ZOOTECNIA, 37., 2000 , Viçosa. Anais... Viçosa: Sociedade Brasileira de Zootecnia, 2000. CD ROM. Nutrição de Ruminantes - 786.

SWATLAND, H.J.; CASSENS, R.G. Inhibition of muscle growth in foetal sheep. Journal of Agricultural Science, v.80, p.503$509,1973$.

TATUM, J.D.; DeWALT, M.S.; LeVALLEY, S.B. et al. Relationship of feeder lamb frame size feedlot gain and carcass yield and quality grades. Journal of Animal Science, v.76, p.435-440, 1998.

WILSON, S.J.; McEWAN, J.C.; SHEARD, P.W.; HARRIS, A.J. Early stages of myogenesis in a large mammal: formation of successive generations of myotubes in sheep tibialis cranialis muscle Journal Muscle Research, v.13, n.5, p.535-550, 1992.

WIGMORE, P.M., STICKLAND, N.C. Muscle development in large and small pig fetuses. Journal Anatomy, v.137, p.235-45, 1983.

YAMAMOTO, S.M.; MACEDO, F.A.F.; ZUNDT, M. et al. Fontes de oleo vegetal na dieta de cordeiros em confinamento. Revista Brasileira de Zootecnia, v.34, n.2, p.703-710, 2005.

ZEOULA, N.M.B.L. Influência da alimentação nas características quantitativas da carcaça e qualitativas da carne de cordeiros Morada Nova. Jaboticabal: Universidade Estadual Paulista, 2002. 65p. Dissertação (Mestrado em Zootecnia) - Universidade Estadual Paulista, 2002.

ZUNDT, M.; MACEDO, F.A.F.; MARTINS, E.N. et al. Desempenho de cordeiros alimentados com diferentes níveis protéicos. Revista Brasileira de Zootecnia, v.31, n.3, p.1307-1314, 2002.

ZUNDT, M.; MACEDO, F.A.F, MARTINS, E.N. et al. Características de carcaça de cordeiros terminados em confinamento, com dietas contendo diferentes níveis protéicos. Ciência Rural, v.33, n.3, p.565-571, 2003. 